Results We identified 111 patients between 2017 and 2020, 57 patients required a drip (43 NCR, $14 \mathrm{CLV})$, and 54 patients were managed with prn medications. Median NIHSS was higher in the drip group (18, IQR 13-21) vs. 15.5 (IQR $9-20), p$ value $=0.03$. There was no significant difference in the rate of good clinical outcome defined as mRs of $0-2$ or return to baseline $(47.4$ vs $61.1 \%, \mathrm{p}$ value $=0.18)$; rate of any HI $(29.8 \%$ vs. $27.8 \%$, p value $=0.84)$ and $\operatorname{siCH}(5.3 \%$ vs $0 \%$, $\mathrm{p}$ value $=0.24$ ) between the two groups (drip vs. no drip). When comparing the NCR to CLV groups, median ROBP was significantly shorter in the CLV group, $5 \mathrm{~min}$ (IQR 0.75-7) vs. $17 \mathrm{~min}$ (IQR 6-35), $\mathrm{p}$ value $=0.003$; and total duration of time outside the pre-specified BP range was $37.5 \mathrm{~min}$ (IQR $19-120)$ vs. $118 \mathrm{~min}(57-227)$ consecutively, $\mathrm{p}$ value $=0.045$. HT rates $(14.3 \%$ vs. $34.9 \%$, p value $=0.19)$, sICH rates $(0$ vs. $7 \%, \mathrm{p}$ value $=0.57)$, and rate of good clinical outcome $(64.3 \%$ vs. $41.9 \%, \mathrm{p}$ value $=0.22$ ) were similar between the two groups (CLV vs. NCR). In a Multivariate analysis, after adjusting for age, gender, IV tPA administration, drip used and time out of range; NIHSS (OR 0.8, CI 0.70-0.91, p=0.0008) and ROBP $(\mathrm{OR}=1.05$, CI 1.0-1.1, p value $=0.032)$ were independent predictors of good clinical outcome.

Conclusion CLV achieved faster ROBP time and better BP control after MT. Further prospective studies are needed.

Disclosures M. Oliver: None. J. Shawver: None. H. Salahuddin: None. S. Desai: None. S. Zaidi: None. M. Jumaa: None.

\section{E-085 INTERVENTIONAL AND DIAGNOSTIC NEURORADIOLOGY FELLOWSHIP EDUCATION IN THE COVID-19 ERA}

'Z Wilseck*, ${ }^{2} S$ Bamezai, ${ }^{2} \mathrm{~N}$ Novakovic, ${ }^{3} \mathrm{~A}$ Copelan, ${ }^{4} \mathrm{~J}$ Wilseck, ${ }^{1} \mathrm{~A}$ Srinivasan, ${ }^{5} \mathrm{~A}$ Pandey, ${ }^{5} \mathrm{G}$ Thompson, 'J Gemmete, ${ }^{1} \mathrm{~N}$ Chaudhary. ${ }^{1}$ Radiology, University of Michigan Health System, Ann Arbor, Mli ${ }^{2}$ University of Michigan Health System, Ann Arbor, MI; ${ }^{3}$ Radiology, Consulting Radiologists Ltd, Edina, MN; ${ }^{4}$ Radiology, Oakland University William Beaumont Hospital, Royal Oak, MI; Neurosurgery, University of Michigan Health System, Ann Arbor, MI

\subsection{6/neurintsurg-2021-SNIS. 180}

Background and Purpose Given the relatively short duration and multiple facets of education, both interventional and diagnostic neuroradiology fellowships must be used efficiently. As hospital systems utilize resources to provide clinical care of patients with COVID-19, the overall number of elective neurointerventional procedures has been limited and the overall volume of diagnostic imaging has decreased. This article will evaluate and discuss the impact of procedural volumes between two academic centers, the impact on overall diagnostic neuroradiology imaging volume, and the rapid migration to online web-based education and research collaboration on both interventional and diagnostic neuroradiology fellowship experiences in the COVID-19 era.

Material and methods A retrospective review was conducted at two academic tertiary care hospitals to evaluate the change in overall elective and emergent neurointerventional case volume during the COVID-19 pandemic spanning from January 2020 - April 2020 and those volumes were compared to preCOVID-19 case volume during January 2019-April 2019. A single center comparison of annual neurointerventional case numbers during 2019 and 2020 was performed with sub-analyses of case types. Annual diagnostic neuroradiology imaging volume was also evaluated for 2019 and 2020.

Results Both tertiary care academic medical centers experienced a statistically significant decrease in the number of elective neurointerventional cases between March - April 2019 and March - April 2020. There was also a statistical different decrease in elective case volume during the fourth quarter of the 2020 academic year (April - June). A statistically significant decrease in case volume was not seen in the setting of thrombectomy for acute ischemic stroke. A statistically significant decrease in aneurysmal subarachnoid hemorrhage (aSAH) case volume was only seen at site \#2. Between 2019 and 2020 there was a $32 \%$ drop in neuroradiology case volume during the fourth quarter of the 2020 academic year (April June). Similar sizable decreases in diagnostic neuroradiology case volume were not appreciated in the remaining quarters of 2019 and 2020.

Conclusion Elective neurointerventional case numbers decreased as a result of the COVID-19 pandemic, particularly impacting the fourth quarter of the 2019-2020 academic year (April - June). Similarly, there was a sizable decrease in diagnostic neuroradiology case volume during this same time period. The decrease in procedural and diagnostic neuroradiology cases raised concerns regarding the educational experience for both diagnostic and neurointerventional radiology fellows. However, as a result of decreased case volume, fellowship education was rapidly augmented with a wide variety of online webinars, lectures, case conferences, and research collaborations.

Disclosures Z. Wilseck: None. S. Bamezai: None. N. Novakovic: None. A. Copelan: None. J. Wilseck: None. A. Srinivasan: None. A. Pandey: None. G. Thompson: None. J. Gemmete: None. N. Chaudhary: None.

\section{E-086 HUMAN INTRACEREBRAL HEMORRHAGE (ICH): EARLY HEMOLYSIS/ERYTHRYOLYSIS, IRON OVERLOAD, PERIHEMATOMAL EDEMA \& SURVIVING WHITE MATTER; TRANSLATIONAL EVIDENCE FOR BRAIN TISSUE INJURY MARKERS ON MRI}

${ }^{1} \mathrm{~N}$ Novakovic, ${ }^{2} \mathrm{Z}$ Wilseck*, ${ }^{2} \mathrm{~T}$ Chenevert, ${ }^{3} \mathrm{G} X \mathrm{X},{ }^{3} \mathrm{R}$ Keep, ${ }^{3} \mathrm{~A}$ Pandey, ${ }^{2} \mathrm{~N}$ Chaudhary. ${ }^{1}$ University of Michigan Health System, Ann Abor, MI; ${ }^{2}$ Radiology, University of Michigan Health System, Ann Abor, Ml; ${ }^{3}$ Neurosurgery, University of Michigan Health System, Ann Abor, MI

\subsection{6/neurintsurg-2021-SNIS. 181}

Objective Intracerebral Hemorrhage (ICH) in humans has not benefited from any translation from the benchside to the bedside in terms of established therapy or prognostic markers to guide treatment. ICH continues to inflict devastating neurological consequences. There is gatheringevidence for some MRI parameters to reflect the amount of iron that leaches out of the hematoma to cause neurotoxicity. We examine the role of early erythrolysis within the hematoma as determined by MRI in ICH patients and its role in predicting iron overload to the surrounding tissues and its effects on the surrounding white matter based on MRI signal quantification.

Methods All patients recruited in the study since 2017 were included in the analysis. A total of 15 patients were prospectively enrolled into the NIH funded study since 2017 with informed consent and local IRB approval. The patients had MRIs at day $1 \& 3$, termed as early, day 14 designated as subacute period and day 30 as the late period. All MRIs were performed on a 3 Tesla MRI. Analysis and measurements were performed on $\mathrm{T} 2 *$ maps, relaxivity (R2*) maps, fractional anisotropy (FA) maps, and edema extent on T2 or FLAIR sequences. Individual measured parameter was then 
correlated with reference to measured iron levels in the surrounding brain tissue and the extent of erythrolysis/hemolysis within the hematoma.

Results Iron overload (IO) to the brain tissue surrounding the hematoma from a given hematoma correlates positively with increasing size of the hematoma. The erythrolysis/hemolysis proportion increases significantly with time up to day 14 , evident in almost each individual patient. The IO also positively correlates with proportion of early erythrolysis/hemolysis at days $14 \& 30$. Perihematomal proportion of white matter survival positively correlates with hematoma volume and hemolysis volume at \&lt day 3 , day $14 \&$ day 30 .

Conclusion Our preliminary study suggests that MRI can reliably assess the proportion of erythrolysis that occurs within the hematoma. Our study also shows that there is reliability in measurement of tissue toxicity markers like iron that leaches out from the hematoma into surrounding tissue (IO) and its correlation with proportional lysis within the hematoma over a period of 1 month. In addition, hemolysis/eryhtrolysis percentage within hematoma and its size correlates with surviving white matter tissue around the hematoma. The above mentioned parameters could become objective markers of tissue injury secondary to an $\mathrm{ICH}$ and once validated further in a larger human sample could further inform the assessment of multiparametric prognostic indices of human ICH on MRI.

Disclosures N. Novakovic: None. Z. Wilseck: None. T. Chenevert: None. G. Xi: None. R. Keep: None. A. Pandey: None. N. Chaudhary: None.

\section{E-087 NEUROFORM ATLAS STENT-ASSISTED COILING: JAILING VS RE-CROSSING TECHNIQUES}

${ }^{1}$ A Khaldi ${ }^{*},{ }^{1} \mathrm{P}$ Williams, ${ }^{2} \mathrm{~A}$ Jadhav, ${ }^{3} \mathrm{R}$ Hanel, ${ }^{4} \mathrm{D}$ Frei, ${ }^{5} \mathrm{~S}$ Hetts, ${ }^{6} \mathrm{O}$ Zaidat, ${ }^{7} \mathrm{~B}$ Jankowitz. ${ }^{1}$ Neurosurgery, Wellstar Medical Group, Marietta, GA; ${ }^{2}$ Division of Neurological Surgery, Barrow Neurological Institue, Phoenix, AZ; ${ }^{3}$ Neurosurgery, Baptist Neurological Institute, Jacksonville, FL; ${ }^{4}$ Radiology Imaging Associates, Swedish Medical Center, Englewood, CO ${ }^{5}$ Interventional Neuroradiology, University of California San Francisco, San Francisco, $C A_{i}$ ${ }^{6}$ Neuroscience, Bon Secours Mercy, Toledo, OH; ${ }^{7}$ Neurosurgery, Cooper University Hospital, Camden, NJ

\subsection{6/neurintsurg-2021-SNIS.182}

Introduction Stent-assisted coil embolization of cerebral aneurysms can be performed using one of multiple techniques. Jailing is the ability to trap microcatheter in the aneurysm while laying a stent across the neck of the aneurysm followed by coiling the aneurysm. Crossing technique refers to placing a microcatheter through the interstices of the stent and then into the aneurysm in order to treat it. Neuroform Atlas stent can be delivered via a 0.0165 inch inner diameter microcatheter which would allow for easier placement of two microcatheters simultaneously and hence a more efficient trapping technique.

Method Over 298 patients were treated in multiple centers with Neuroform Atlas Stent-assisted coiling of aneurysms. The treating physicians decided on the technique of treatment including jailing, crossing, or others (jailing followed by recrossing, stenting followed by re-crossing at a different time, or unknown). Patients' demographics, shape, dimension, and location of the aneurysm, as well as the number of coils and procedure duration were analyzed ad hoc. Intra-procedure complications of the different techniques were also recorded. In addition, a 12-month follow up angiogram (248/298) was performed, and Raymond score was evaluated by core laboratory.

Results Similar characteristics were observed between the two groups (jailing and re-crossing) in the shape, dimensions, and location of the aneurysms. There was also no increase in the number of coils used or duration of the procedure between the two different techniques. Procedures related adverse events and device related adverse events were similar between the 2 groups. A higher proportion of complete occlusion and a lower proportion of residual aneurysm (2.7\%) were observed in the jailing group, compared with the re-cross group (7.3\%). However, the difference is not statistically significant.

Conclusion Jailing technique might present an alternative to re-crossing the Neuroform Atlas Stent without a significant increase in complications and it may decrease the chance of aneurysm recurrence at 12 months.

Disclosures A. Khaldi: None. P. Williams: None. A. Jadhav: None. R. Hanel: 1; C; Medtonic, Stryker, Cerenovus, Microvention. 2; C; Stryker, Medtronic, Cerenovus, Microvention. 4; C; Neurvana, Elum, EndoStream, Three Rivers Medical Inc, Cerebrotech, In NeuroCO.. 6; C; Scientific Advisor, MIVI, Scientific Advisor, Elum, Scientific Advisor, Three Rivers Medical. D. Frei: 2; C; Stryker, Siemens. 3; C; Stryker, Penumbra, Philips, Genetech, Siemens, VIZ AI. 6; C; Medical Advisor for Memory Medical. S. Hetts: 2; C; Microvention. 6; C; Core Lab services for Stryker and Micro Vention Terumo. O. Zaidat: 1; C; Stryker, Medtronic, Cerenovus, Penumbra, Genetech. 2; C; Cerenovus, Stryker, Penumbra, Medtronic. 6; C; Ownership in Galaxy Therapeutics LLC, Endovascular Committee co chair of NIH StrokeNet Consortium. B. Jankowitz: 2; C; Stryker, Medtronic.

\section{E-088 VISUALIZATION OF THE ANTERIOR TEMPORAL ARTERY AS A PREDICTOR OF OUTCOME AFTER MECHANICAL THROMBECTOMY}

${ }^{1} \mathrm{~J}$ Parish*, ${ }^{2} \mathrm{~W}$ Stetler, ${ }^{3} \mathrm{D}$ Strong, ${ }^{3} \mathrm{~T}$ Prasad, ${ }^{3} \mathrm{~J}$ Rhoten, ${ }^{4} \mathrm{R}$ Karamchandani, ${ }^{5} \mathrm{~J}$ Clemente, ${ }^{5} \mathrm{G}$ Defilipp, ${ }^{5} \mathrm{~A}$ Hines, ${ }^{2} \mathrm{~J}$ Bernard, ${ }^{3} \mathrm{~A}$ Asimos. ${ }^{1}$ Neurological Surgery, Atrium Health, Charlotte, NC; ${ }^{2}$ Carolina Neurosurgery and Spine Associates, Charlotte, NC; ${ }^{3}$ Atrium Health, Charlotte, NC; ${ }^{4}$ Neurology, Atrium Health, Charlotte, NC; ${ }^{5}$ Charlotte Radiology, Charlotte, NC

\subsection{6/neurintsurg-2021-SNIS.183}

Introduction The anterior temporal artery (ATA) visualization on Computerized Tomography Angiography (CTA) has been previously associated with good outcomes in middle cerebral artery (MCA) occlusions, but not in the context of recanalization after mechanical thrombectomy. We hypothesized that independent functional outcome at 90 days would be greater for MCA occlusion patients with a visualized ATA on CTA who achieved Thrombolysis in Cerebral Infarction (TICI) 2b or greater recanalization.

Methods We conducted a retrospective cohort study of patients with acute ischemic stroke who underwent mechanical thrombectomy. A neuroradiologist blinded to patient outcomes confirmed the MCA as the most proximal site of occlusion on CTA and assessed for visualization of the ATA. TICI $2 \mathrm{~b}$ or greater revascularization scores were confirmed by neurointerventionalists blinded to patient outcomes. Estimates of the volume of ischemic core and penumbral regions from CT perfusion were calculated with the use of RAPID software (iSchemaView). Ninety-day mRS scores were obtained via telephone utilizing a structured questionnaire. 